\title{
Pattern of Blood Stream Infections within Neonatal Intensive Care Unit, Suez Canal University Hospital, Ismailia, Egypt
}

\author{
Rania Mohammed Kishk, ${ }^{1}$ Mohamed Fouad Mandour, ${ }^{2}$ Rasha Mohamed Farghaly, ${ }^{3}$ \\ Ahmed Ibrahim, ${ }^{4}$ and Nader Attia Nemr ${ }^{5}$ \\ ${ }^{1}$ Microbiology and Immunology Department, Faculty of Medicine, Suez Canal University, Ismailia, Egypt \\ ${ }^{2}$ Clinical Pathology Department, Faculty of Medicine, Suez Canal University, Ismailia, Egypt \\ ${ }^{3}$ Occupational Medicine Department, Faculty of Medicine, Suez Canal University, Ismailia, Egypt \\ ${ }^{4}$ Pediatric Department, Faculty of Medicine, Suez Canal University, Ismailia, Egypt \\ ${ }^{5}$ Endemic and Infectious Disease Department, Faculty of Medicine, Suez Canal University, Ismailia, Egypt
}

Correspondence should be addressed to Rania Mohammed Kishk; rankishk@yahoo.com

Received 24 June 2014; Revised 20 September 2014; Accepted 29 September 2014; Published 20 October 2014

Academic Editor: Todd R. Callaway

Copyright (C) 2014 Rania Mohammed Kishk et al. This is an open access article distributed under the Creative Commons Attribution License, which permits unrestricted use, distribution, and reproduction in any medium, provided the original work is properly cited.

\begin{abstract}
Introduction. Blood stream infection (BSI) is a common problem of newborn in neonatal intensive care units (NICUs). Monitoring neonatal infections is increasingly regarded as an important contributor to safe and high-quality healthcare. It results in high mortality rate and serious complications. So, our aim was to determine the incidence and the pattern of BSIs in the NICU of Suez Canal University Hospital, Egypt, and to determine its impact on hospitalization, mortality, and morbidity. Methods. This study was a prospective one in which all neonates admitted to the NICUs in Suez Canal University hospital between January, 2013 and June 2013 were enrolled. Blood stream infections were monitored prospectively. The health care associated infection rate, mortality rate, causative organism, and risk factors were studied. Results. A total of 317 neonates were admitted to the NICU with a mortality rate of $36.0 \%$. During this study period, 115/317 (36.3\%) developed clinical signs of sepsis and were confirmed as BSIs by blood culture in only 90 neonates with 97 isolates. The total mean length of stay was significantly longer among infected than noninfected neonates $(34.5 \pm 18.3$ and $10.8 \pm 9.9$ days, resp., $P$ value $<0.001)$. The overall mortality rates among infected and noninfected neonates were $38.9 \%$ and $34.8 \%$, respectively, with a significant difference. Klebsiella spp. were the most common pathogen (27.8\%) followed by Pseudomonas (21.6\%) and Staphylococcus aureus (15.4\%). Conclusion. The rate of BSIs in NICU at Suez Canal University Hospital was relatively high with high mortality rate $(36.0 \%)$.
\end{abstract}

\section{Introduction}

Infection rates are standard indicators of quality and safety in all healthcare settings all over the world [1]. The challenge of infection control policies and procedures is always to decrease the frequency of healthcare-associated infections (HAI). Consequently determining infection rates through a surveillance program is central step in both identifying problems and evaluating the implementation of any program [2]. Healthcare-associated infections are associated with increased morbidity, mortality, and economic burden (either direct or indirect) [3]. More than 2,000,000 HAI in children and adults were reported in the United States, resulting in prolonged hospitalization with increased healthcare costs each year $[4,5]$.

Healthcare-associated infections in the neonatal intensive care units (NICUs) are affected by many factors as endemic microbial flora, clinical techniques, and antibiotic stewardship policies. Consequently, neonatal infections became more than a challenge for pediatricians [6].

Blood stream infections (BSIs) caused by commensal species play important roles in nosocomial infections in the NICU, which poses difficulties in determining true pathogens from contaminants [7]. It also served as the single most important type of infection because of their high frequency (59\%) and potential life-threatening consequences [8]. 
Monitoring neonatal infections is increasingly regarded as an important contributor to safe and high-quality healthcare [9]. However, only few studies described BSIs in Egyptian NICUs. The objectives of this study were to determine the incidence and the pattern of BSIs in the NICU of Suez Canal University Hospital, Ismailia, Egypt, and to determine its impact on hospitalization, mortality, and morbidity among those critical age group patients.

\section{Methods}

2.1. Setting. The NICU at Suez Canal University Hospital is a referral unit that is supplied with 26 incubators. The unit receives newborns from the obstetric department, several hospitals, and healthcare centers in Suez Canal region. A trained infection control link nurse is available at the unit at all time with average 1 nurse for 2 neonates. A detailed written policies and procedures for hand hygiene are made clear in the unit. Disinfectants dispensers filled with alcohol $70 \%$ were provided at hand-wash stations; however, clean towels for hand-drying were not sufficiently available. Sterile and clean nonsterile gloves were used during neonatal care according to the followed guidelines. No routine surveillance cultures were taken and however sometimes used when an outbreak was suspected.

2.2. Study Design and Patient Population. This study was a prospective one in which all neonates admitted to the NICUs in Suez Canal University Hospital between January 2013 and June 2013 were enrolled in the study. During these 6 months, 317 neonates were admitted to the unit with no evidence of infection by clinical assessment at the time of admission. Blood stream infections were monitored prospectively by the attending pediatrician staff and infection control specialist.

2.3. Definitions. Subjects who had no infection and/or were not in the incubation period at presentation and who developed infection more than 72 hours after hospitalization were considered to have nosocomial infection. The diagnosis of nosocomial blood stream infection was made by clinical findings, laboratory findings, and hemoculture. Clinical findings including hypothermia, hyperthermia, apnea, bradycardia, circulatory disorder, lethargy, hypotonia, and feeding difficulty and laboratory findings including leukocytosis, leucopenia, thrombocytopenia, a ratio of immature/mature neutrophils $>0.25$, and a C-reactive protein $(\mathrm{CRP})$ value of $>1 \mathrm{mg} / \mathrm{dL}$ were considered to be significant.

2.4. Laboratory Methods. For each neonate, two blood samples per patient ( $1 \mathrm{~mL}$ each) were drawn under sterile conditions at least $30 \mathrm{~min}$ apart from each other. All blood cultures were processed by the Microbiology Laboratory, Faculty of Medicine, Suez Canal University. Blood culture bottles, especially for neonates, were incubated for ten days at $37^{\circ} \mathrm{C}$ and subcultured every other day to sheep blood agar, chocolate agar, MacConkey agar, and mannitol salt agar plates (oxoid) and incubated at $37^{\circ} \mathrm{C}$ for $24-48$ hours. Isolates of bacteria were identified by conventional biochemical and
TABLE 1: General characteristics of the admitted neonates $(n=317)$ during 6-month period.

\begin{tabular}{lc}
\hline Characteristics & Number (\%) \\
\hline Gender & \\
Male & $194(61.2 \%)$ \\
Female & $123(38.8 \%)$ \\
Birth weight & \\
$\quad<1000$ gm. & $104(32.8 \%)$ \\
1000 gm $-<1500$ gm & $143(45.1 \%)$ \\
$1500-2500$ gm & $48(15.1 \%)$ \\
$>2500$ gm & $22(7 \%)$ \\
Discharge status & \\
Alive & $203(64.0 \%)$ \\
Died & $114(36.0 \%)$ \\
\hline
\end{tabular}

serological methods according to the Clinical Laboratory Standard Institute (CLSI) criteria [10].

2.5. Susceptibility Testing. The antibiotic susceptibility for isolated pathogens was determined on Muller Hinton (oxoid) by Kirby-Bauer disk diffusion method and interpreted according to the National Committee for Clinical Laboratory Standards breakpoint values [11]. A BSI was defined as isolation of at least one positive peripheral-blood culture, whereas in cases of coagulase-negative staphylococci infections two positive blood cultures were required [12].

2.6. Statistical Analysis of Data. Statistical Package for Social Sciences (SPSS) for Windows 15 program was used for statistical analysis of the results obtained in the study. Demographic properties in the study were assessed by "descriptive" statistical analysis. "Unpaired" $t$-test was used for comparison of groups.

\section{Results}

During the 6-month period started at January 2013 till June 2013, a total of 317 neonates were admitted to the NICU in Suez Canal University; of these neonates 114 (36.0\%) died (Table 1).

During this study period, 115 (36.3\%) developed clinical signs of sepsis and confirmed by blood culture as blood stream infection in only 90 neonates $(78.26 \%)$ with 97 isolates. The general characteristics of the infected neonates are shown in Table 2. The most common reason for hospitalization was preterm delivery in patients with nosocomial infection. The other reasons for hospitalization included meconium aspiration syndrome, transient tachypnea or asphyxia, congenital cardiac disease, and congenital metabolic diseases.

In $43 \%$ of the patients who had a positive culture, there was no problem in antenatal history, but $57 \%$ suffered from preeclampsia, eclampsia, hypothyroidism, and cardiac problems in antenatal period.

During this 6-month period of study, we noticed that the highest rate of death was in January although it has the lowest admission rate (29/47). The highest rate of admission was 
TABLE 2: Characteristics of the infected neonates $(n=90)$ within 6month period.

\begin{tabular}{lc}
\hline Characteristics & Number (\%) \\
\hline Gender & $59(65.6 \%)$ \\
Male & $31(34.4 \%)$ \\
Female & \\
Birth weight & $49(54.4 \%)$ \\
$\quad<1000$ gm. & $30(33.3 \%)$ \\
1000 gm-<1500 gm & $11(12.3 \%)$ \\
$1500-2500$ gm & $0(0)$ \\
$>2500$ gm & \\
Discharge status & $55(61.1 \%)$ \\
Alive & $35(38.9 \%)$ \\
Died &
\end{tabular}

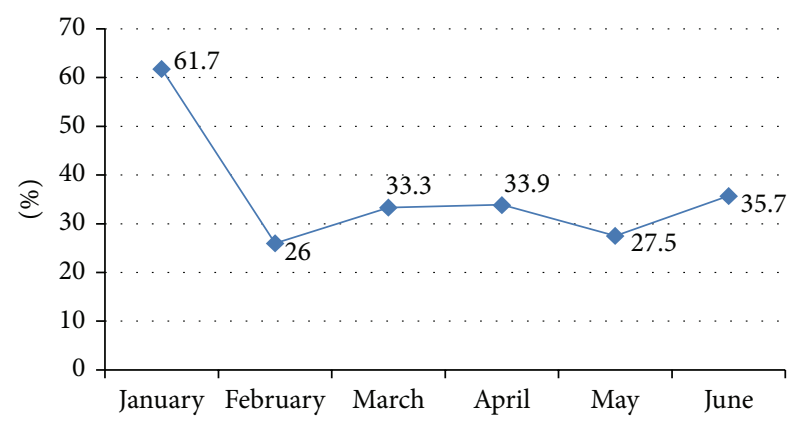

FIGURE 1: Frequency (\%) of blood stream infections among admitted neonates during the study.

in March followed by April and June (57 versus 56 and 56 neonates, resp.) (Figure 1).

The most common clinical findings of blood stream infection were found to be apnea and bradycardia (45\%), hypo/hyperthermia was found in $27 \%$, circulatory disorder was found in $20 \%$, and feeding intolerance was found in $17 \%$. Laboratory findings included leukocytosis (64\%), leucopenia (27\%), thrombocytopenia (50\%), an immature/mature neutrophils ratio of $>0.25(47 \%)$, and increased CRP $(77 \%)$.

3.1. Microbial Causes of Blood Stream Infection. The identified microorganisms in blood cultures are shown in Figure 2. A total of 65 isolates $(67 \%)$ of 97 recovered isolates were Gram negative organisms, 30 isolates (31\%) were Gram positive organisms, and only 2 isolates were Candida. Klebsiella pneumoniae were the most common pathogen accounting for $27.8 \%$ of the total isolates, followed by Pseudomonas (21.6\%) and Staphylococcus aureus (15.4\%). Although two bacterial sepsis episodes were observed more frequently in preterm babies, the difference was not statistically significant. Positive hemoculture was accompanied by pneumonia (35\%), meningitidis (15.6\%), or urinary infection (5\%).

3.2. Complications of Infection. All infected neonates were on peripheral line cannulation or umbilical vein cannulation. Out of 90 infected neonates, the device related infection was significantly related to the length of the stay as $56.7 \%$ (51 neonates) were staying for more than 1 week in the incubator, $37.8 \%$ (34 neonates) for 2 days till up to 7 days, and only $5.5 \%$ (5 neonates) for 1 day.

3.3. Antibiotic Susceptibility. Aminoglycosides were found to be as efficient as third generation cephalosporins for Klebsiella species. Pseudomonas species were found to be highly sensitive to ciprofloxacin, imipenem, meropenem, and piperacillin-tazobactam and resistant to tobramycin, cefotaxime, and ceftriaxone as shown in Table 3.

Serratia species were resistant totobramycin, ciprofloxacin, imipenem, meropenem cefotaxime, and ceftriaxone. Acinetobacter spp. were found to be resistant to penicillin, aminoglycosides, and all cephalosporins.

Staphylococcus aureus was found to be sensitive to amikacin, vancomycin, teicoplanin, and linezolid with the highest degree and to oxacillin with the lowest degree. Coagulase negative staphylococci were found to be sensitive to vancomycin, linezolid, and teicoplanin. All the strains were resistant to ampicillin and penicillin (Table 3 ).

\section{Discussion}

Neonatal infections are an important cause of mortality and morbidity worldwide. In the World Health Organization 2000-2003 report, neonatal sepsis and pneumonia were responsible for about 1.6 million deaths each year, mainly in resource-poor countries [13].

In the present study, the total admitted neonates were 317; BSIs are considered one of the most important infections in our hospital (representing 28.4\%) because of their potential life-threatening consequences that may end by death. Ho and his coworkers [8] considered BSIs the most common source of infection as they represented $59.0 \%$ and $5.6 \%$ of these were catheter-related [8]. On the other hand, in a recent study in Egypt, pneumonia was the most frequently occurring infection (11.3\%) followed by blood stream infection $(8.8 \%)$ in NICU [14] which comes in agreement with the two Brazilian studies $[15,16]$. However, in developed countries the distribution of nosocomial infections in neonates is different, with BSI predominating, followed by pneumonia $[15,17,18]$. Most cases of BSIs in the NICU are associated with indwelling vascular catheters $[19,20]$. Other risk factors may be associated with BSIs in the NICU as lipids administration, low birth weight, respiratory disease, catheter hub colonization, blood sampling for central venous catheters, and use of $\mathrm{H} 2$ blockers [21]. In another recent study for the prevalence of different nosocomial infections, Van der Zwet et al. studied one hundred and ninety-one neonates which developed 264 infections. They discovered blood stream infection is the most common nosocomial infection in NICUs $(N=138)$ followed by pneumonia $(N=69)$ [7].

Differences in BSIs rates between studies may be partially explained by the methodology and definitions used, especially in relation to the definitions of infection. The present study included patients only with microbiologically confirmed infections (90 neonates) although 115 neonates 
TABLE 3: Antibiotic sensitivity of Gram negative organisms.

\begin{tabular}{|c|c|c|c|c|c|c|}
\hline & $\begin{array}{c}\text { Klebsiella }(n: 27) \\
\text { Resistance }(n)\end{array}$ & $\begin{array}{c}\text { Pseudomonas ( } n: 21) \\
\text { Resistance }(n)\end{array}$ & $\begin{array}{l}\text { Serratia }(n: 12) \\
\text { Resistance }(n)\end{array}$ & $\begin{array}{c}\text { E. coli }(n: 2) \\
\text { Resistance }(n)\end{array}$ & $\begin{array}{l}\text { Proteus }(n: 1) \\
\text { Resistance }(n)\end{array}$ & $\begin{array}{c}\text { Acinetobacter }(n: 1) \\
\text { Resistance }(n)\end{array}$ \\
\hline \multicolumn{7}{|c|}{ Beta-lactams } \\
\hline Ampicillin & 20 & 3 & 10 & 2 & 1 & 1 \\
\hline Piperacillin-tazobactam & 18 & 1 & 12 & 1 & 0 & 1 \\
\hline Sulperazone & 17 & 0 & 9 & 1 & 0 & 1 \\
\hline Cefotaxime & 15 & 21 & 12 & 1 & 1 & 1 \\
\hline Ceftriaxone & 19 & 18 & 12 & 2 & 1 & 1 \\
\hline Ceftazidime & 18 & 3 & 12 & 1 & 1 & 1 \\
\hline Imipenem & 1 & 1 & 11 & 1 & 1 & 0 \\
\hline Meropenem & 1 & 1 & 10 & 1 & 1 & 0 \\
\hline \multicolumn{7}{|c|}{ Non-beta-lactam } \\
\hline Amikacin & 15 & 1 & 10 & 0 & 0 & 1 \\
\hline Gentamycin & 3 & 20 & 8 & 2 & 1 & 1 \\
\hline Tobramycin & 5 & 21 & 4 & 2 & 1 & 1 \\
\hline Ciprofloxacin & 5 & 21 & 9 & 2 & 1 & 1 \\
\hline
\end{tabular}

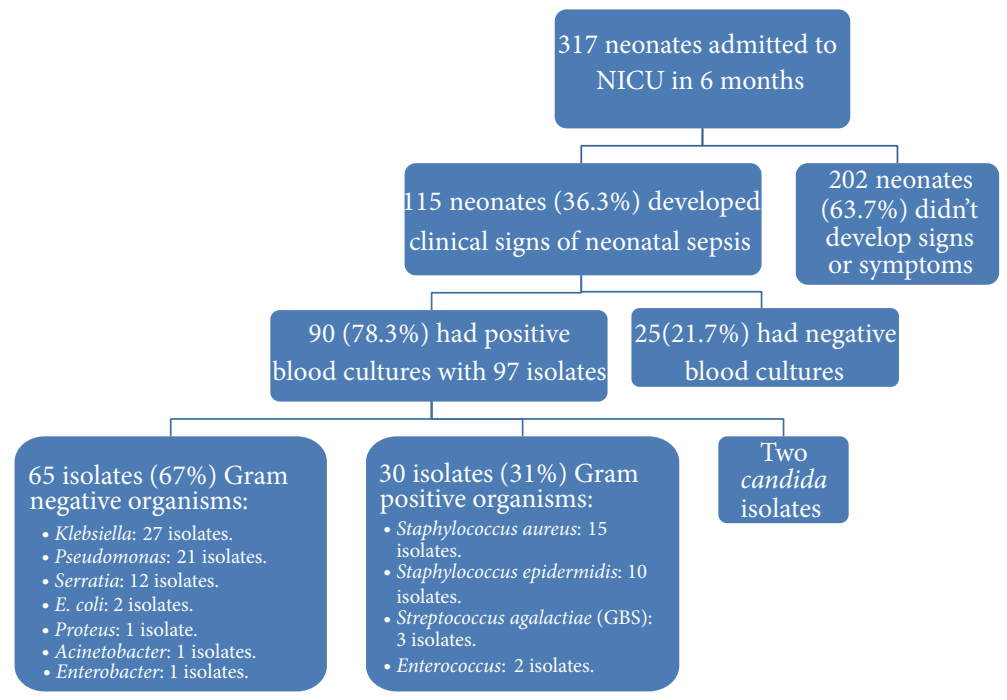

FIGURE 2: Study enrollment chart and pathogens recovered from neonatal blood culture.

developed clinical signs of sepsis; this may partly explain why the infection rate appeared lower than in some other reports from developing countries, which included patients with clinical evidence of infection only [16].

In the present study, 115 neonates (36.3\%) developed clinical signs of neonatal sepsis; 90 neonates $(78.26 \%)$ of 115 neonates had positive blood cultures with 97 isolates. Van der Zwet et al. [7] and his team in 2005 mentioned that at least $25 \%$ of clinically diagnosed septic episodes are culturenegative [7]. This may be due to many factors as concurrent use of antibiotics and suboptimal sample volumes.

The incidence of neonatal sepsis is inversely proportional to birth weight, with incidences of sepsis in infants of birth weight $<1000 \mathrm{~g}$ of $54.4 \%$, compared with $33.3 \%$ in those weighing $1000-<1500 \mathrm{~g}$ and $12.3 \%$ in those weighting $1500-2500 \mathrm{gm}$ and $0 \%$ in those neonates above
2500 gm. These results are compatible with Kaufman and Fairchild [22] who reported that $20 \%$ of very-low-birthweight $(<1500 \mathrm{~g})$ preterm infants experience a serious systemic infection and even with advanced neonatal intensive care and antimicrobials, a threefold higher mortality was reported for these infants who develop sepsis than their counterparts without sepsis during their hospitalization [22].

In our study, Gram negative pathogens were responsible for most neonatal BSIs representing 67\% (65 isolates out of 97 isolates). Klebsiella pneumoniae and Pseudomonas are the cause of nearly half of infections (73.8\%) followed by Serratia (12 isolates), E. coli (2 isolates), Proteus (1 isolate), Acinetobacter (1 isolate), and Enterobacter (1 isolate). In another descriptive hospital-based study carried out for 12 months in the NICU of the Mansoura University Children's 
Hospital, the investigators reported that Klebsiella spp. were the most frequently isolated organisms followed by $E$. coli [14] which is consistent with the results of previous Egyptian studies $[23,24]$.

In 2002, Huang and his coworkers described Acinetobacter baumannii as nosocomial pathogens in adults, being also responsible for infections in neonates hospitalized in ICUs, causing pneumonia. It is a ubiquitous microorganism implicated in a number of outbreaks in ICUs [25]. One year later, in 2003, Villegas and Hartstein [26] mentioned that most of these outbreaks have been traced to environmental sources, such as mechanical ventilation equipment and air conditioners [26].

In comparison with previous studies, CoNS is the predominant source of infection in NICU in the United States and Asian countries [27, 28] which is quite different from the community-acquired setting. This is not compatible with our results as the most predominant Gram positive organism was Staphylococcus aureus representing 15\% of isolated Gram positive organisms. S. aureus is the most common pathogen causing pustulosis and cellulitis in neonates. The presence of virulence factors such as the Panton-Valentine leukocidin is thought to contribute to the pathogen's ability to cause skin and soft tissue infections [29]. In addition to the underdeveloped epidermis and frequent breeches in skin integrity due to intravenous catheters, blood draws and heel sticks place preterm neonates at risk of infection [30]. With advances in our NICU, the survival rates, hospital stay, and number of invasive procedures have increased. This might be part of the reason why $S$. aureus became the dominant Gram positive pathogen during the last decade.

By contrast, in western countries, Gram positive bacteria have predominated in most studies with CoNS accounting for $57 \%$ of all nosocomial infections in Australia and Gram positive bacteria were the leading causes of nosocomial infections (70\%) in North America, again with CoNS predominating (accounting for $48 \%$ of infections) [31]. In Europe, most of nosocomial infections (76.4\%) were caused by Gram positive bacteria, with CoNS being the most frequently occurring pathogens [18]. Even in Brazil, CoNS were the most frequent nosocomial pathogens, accounting for $41.6 \%$ of isolates [15].

On the other hand, Ho et al. [8] concluded that BSIs and skin/soft tissue infections caused by commensal species play important roles in healthcare-associated infections in the NICU. An increased incidence of $S$. aureus and A. baumannii infection and a decreased number of CoNS infections were observed. In another recent study, of blood stream infections, $59 \%$ were caused by CoNS [7].

Out of 97 isolates, there were only 2 isolates diagnosed as candidal infection. Candida infections are a common cause of late-onset sepsis in the NICU and are associated with significant mortality and neurodevelopmental impairment [32]. One of the most important reasons in managing candidal infection in our NICU is the use of prophylactic fluconazole in very-low-birth-weight infants to prevent invasive candidiasis. The rationale for this strategy is to prevent fungal colonization in high-risk infants and reduce the invasiveness of the disease [33].
The difference in the species distribution in our study compared with others may partly be attributable to many factors as the gestational age and frequency of use of invasive devices. However, there are many structural factors that may also have affected the pattern of infections as ambient temperature and humidity conditions and the lack of automatic preparation of total parenteral nutrition.

We also found that the mean hospital stay is significantly longer in infected than noninfected neonates. This is consistent with many previously reviewed studies from developing as well as developed countries [16, 34-37]. As all infected neonates were found to 8 be on peripheral line or umbilical vein cannulation, we recommend strict measure for 9 elimination of this line cannulation which would produce an overall great improvement 10 in this high sepsis rate.

Priority should be given to periodic infection surveillance for NICUs, so that standardized infection rates can be used as a benchmark to drive improvements in the quality and safety of care. We recommend also local infection surveillance on NICUs in Ismailia regarding all nosocomial infections which is important to decrease the mortality rate and also to direct rational antimicrobial prescribing. Training programs on the standard precautions, personal protective equipment, and clinical samples collection and transport were implemented in our unit regularly. NICUs should also receive regular reports of antimicrobial susceptibility data from their infection control microbiology laboratory.

\section{Research Directions}

Research directions are as follows:

(i) to assess the awareness, approach, and practice of all healthcare workers in the NICUs. This will be followed by training programs for all the stuff members,

(ii) to study the infection rate and device associated infection in Ismailia NICUs not only Suez Canal University Hospital as a multicenter study.

\section{Conflict of Interests}

The authors declare there is no potential conflict of interests.

\section{References}

[1] D. Pittet, "Infection control and quality health care in the new millenium," The American Journal of Infection Control, vol. 33, no. 5, pp. 258-267, 2005.

[2] G. Ducel, J. Fabry, and L. Nicolle, Eds., Prevention of HospitalAcquired Infections. A Practical Guide, World Health Organization, Geneva, Switzerland, 2nd edition, 2002.

[3] N. R. Payne, J. H. Carpenter, G. J. Badger, J. D. Horbar, and J. Rogowski, "Marginal increase in cost and excess length of stay associated with nosocomial bloodstream infections in surviving very low birth weight infants," Pediatrics, vol. 114, no. 2, pp. 348$355,2004$.

[4] R. P. Wenzel and M. B. Edmond, "Team-based prevention of catheter-related infections," The New England Journal of Medicine, vol. 355, no. 26, pp. 2781-2783, 2006. 
[5] B. J. Stoll, N. I. Hansen, I. Adams-Chapman et al., "Neurodevelopmental and growth impairment among extremely low-birthweight infants with neonatal infection," Journal of the American Medical Association, vol. 292, no. 19, pp. 2357-2365, 2004.

[6] J. W. Gray, "Surveillance of infection in neonatal intensive care units," Early Human Development, vol. 83, no. 3, pp. 157-163, 2007.

[7] W. C. van der Zwet, A. M. Kaiser, R. M. van Elburg et al., "Nosocomial infections in a Dutch neonatal intensive care unit: surveillance study with definitions for infection specifically adapted for neonates," Journal of Hospital Infection, vol. 61, no. 4, pp. 300-311, 2005.

[8] T.-S. Ho, S.-M. Wang, Y.-H. Wu et al., "Long-term characteristics of healthcare-associated infections in a neonatal intensive care unit," Journal of Microbiology, Immunology and Infection, vol. 43, no. 5, pp. 407-415, 2010.

[9] L. M. Mahieu, J. J. de Dooy, A. E. Lenaerts, M. M. Ieven, and A. O. de Muynck, "Catheter manipulations and the risk of catheterassociated bloodstream infection in neonatal intensive care unit patients," Journal of Hospital Infection, vol. 48, no. 1, pp. 20-26, 2001.

[10] M. L. Wilson, M. Mitchell, and A. J. Morris, "Principles and producers for blood culture; approved guidelines," in Proceedings of the Clinical and Laboratory Standards Institute (CLSI'07), pp. 710, 2007.

[11] National Committee for Clinical Laboratory Standards, "Performance standards for antimicrobial susceptibility testing," NCCLS Approved Standard M100-S9, National Committee for Clinical Laboratory Standards, Wayne, Pa, USA, 1999.

[12] N. Macharashvili, E. Kourbatova, M. Butsashvili, T. Tsertsvadze, L.-A. McNutt, and M. K. Leonard, "Etiology of neonatal blood stream infections in Tbilisi, Republic of Georgia," International Journal of Infectious Diseases, vol. 13, no. 4, pp. 499-505, 2009.

[13] K. M. Posfay-Barbe, D. M. Zerr, and D. Pittet, "Infection control in paediatrics," The Lancet Infectious Diseases, vol. 8, no. 1, pp. 19-31, 2008.

[14] F. Abdel-Wahab, M. Ghoneim, M. Khashaba, A.-H. El-Gilany, and D. Abdel-Hady, "Nosocomial infection surveillance in an Egyptian neonatal intensive care unit," Journal of Hospital Infection, vol. 83, no. 3, pp. 196-199, 2013.

[15] J. Y. Kawagoe, C. A. M. Segre, C. R. Pereira, M. F. S. Cardoso, C. V. Silva, and J. T. Fukushima, "Risk factors for nosocomial infections in critically ill newborns: a 5-year prospective cohort study," American Journal of Infection Control, vol. 29, no. 2, pp. 109-114, 2001.

[16] E. Nagata, A. S. J. Brito, and T. Matsuo, "Nosocomial infections in a neonatal intensive care unit: incidence and risk factors," American Journal of Infection Control, vol. 30, no. 1, pp. 26-31, 2002.

[17] A. H. Sohn, D. O. Garrett, R. L. Sinkowitz-Cochran et al., "Prevalence of nosocomial infections in neonatal intensive care unit patients: results from the first national point-prevalence survey," The Journal of Pediatrics, vol. 139, no. 6, pp. 821-827, 2001.

[18] M. Urrea, M. Iriondo, M. Thio et al., "A prospective incidence study of nosocomial infections in a neonatal care unit," American Journal of Infection Control, vol. 31, no. 8, pp. 505-507, 2003.

[19] S. B. Brodie, K. E. Sands, J. E. Gray et al., "Occurrence of nosocomial bloodstream infections in six neonatal intensive care units," Pediatric Infectious Disease Journal, vol. 19, no. 1, pp. $56-65,2000$.
[20] B. J. Stoll and N. Hansen, "Infections in VLBW infants: studies from the NICHD neonatal research network," Seminars in Perinatology, vol. 27, no. 4, pp. 293-301, 2003.

[21] J. Freeman, D. A. Goldmann, N. E. Smith, D. G. Sidebottom, M. F. Epstein, and R. Platt, "Association of intravenous lipid emulsion and coagulase-negative staphylococcal bacteremia in neonatal intensive care units," The New England Journal of Medicine, vol. 323, no. 5, pp. 301-308, 1990.

[22] D. Kaufman and K. D. Fairchild, "Clinical microbiology of bacterial and fungal sepsis in very-low-birth-weight infants," Clinical Microbiology Reviews, vol. 17, no. 3, pp. 638-680, 2004.

[23] M. M. Omar, Nosocomial infections in Kasr El Aini neonatal intensive care unit (NICU) as a university hospital [M.S. thesis], Faculty of Medicine, Cairo University, 2001.

[24] N. Badrawi, M. Bashir, I. Iskander, and D. Saied, "Neonatal infections in NICU of Kasr El-Aini Maternity Hospital," Kasr El-Aini Medical Journal, vol. 11, pp. 180-195, 2005.

[25] Y.-C. Huang, L.-H. Su, T.-L. Wu et al., "Outbreak of Acinetobacter baumannii bacteremia in a neonatal intensive care unit: clinical implications and genotyping analysis," Pediatric Infectious Disease Journal, vol. 21, no. 12, pp. 1105-1109, 2002.

[26] M. V. Villegas and A. I. Hartstein, "Acinetobacter outbreaks, 1977-2000," Infection Control and Hospital Epidemiology, vol. 24, no. 4, pp. 284-295, 2003.

[27] B. J. Stoll, N. Hansen, A. A. Fanaroff et al., "Late-onset sepsis in very low birth weight neonates: the experience of the NICHD Neonatal Research Network," Pediatrics, vol. 110, no. 2, pp. 285291, 2002.

[28] R. Tiskumara, S. H. Fakharee, C. Q. Liu et al., "Neonatal infections in Asia," Archives of Disease in Childhood: Fetal and Neonatal Edition, vol. 94, no. 2, pp. F144-F148, 2009.

[29] L. Saiman, M. O'Keefe, P. L. Graham III et al., "Staphylococcus aureus among postpartum women," Clinical Infectious Diseases, vol. 37, no. 10, pp. 1313-1319, 2003.

[30] A. J. Carey, L. Saiman, and R. A. Polin, "Hospital-acquired infections in the NICU: epidemiology for the new millennium," Clinics in Perinatology, vol. 35, no. 1, pp. 223-249, 2008.

[31] D. Isaacs, "A ten year, multicentre study of coagulase negative staphylococcal infections in Australasian neonatal units," Archives of Disease in Childhood: Fetal and Neonatal Edition, vol. 88, no. 2, pp. F89-F93, 2003.

[32] Y.-C. Huang, T.-Y. Lin, R.-I. Lien et al., "Candidaemia in special care nurseries: comparison of Albicans and Parapsilosis infection," Journal of Infection, vol. 40, no. 2, pp. 171-175, 2000.

[33] P. Manzoni, I. Stolfi, L. Pugni et al., "A multicenter, randomized trial of prophylactic fluconazole in preterm neonates," The New England Journal of Medicine, vol. 356, no. 24, pp. 2483-2495, 2007.

[34] K. R. Shankar, D. Brown, J. Hughes et al., "Classification and risk-factor analysis of infections in a surgical neonatal unit," Journal of Pediatric Surgery, vol. 36, no. 2, pp. 276-281, 2001.

[35] B. J. Stoll, N. Hansen, A. A. Fanaroff et al., "Late-onset sepsis in very low birth weight neonates: the experience of the NICHD neonatal research network," Pediatrics, vol. 110, no. 2, pp. 285291, 2002.

[36] D. M. Ahmed, Epidemiological study of infections in the neonatal surgical unit (NSU) [M.S. thesis], Faculty of Medicine, Cairo University, Giza, Egypt, 2003.

[37] U. A. Mireya, P. O. Martí, K. V. Xavier, L. O. Cristina, M. M. Miguel, and C. M. Magda, "Nosocomial infections in paediatric and neonatal intensive care units," Journal of Infection, vol. 54, no. 3, pp. 212-220, 2007. 

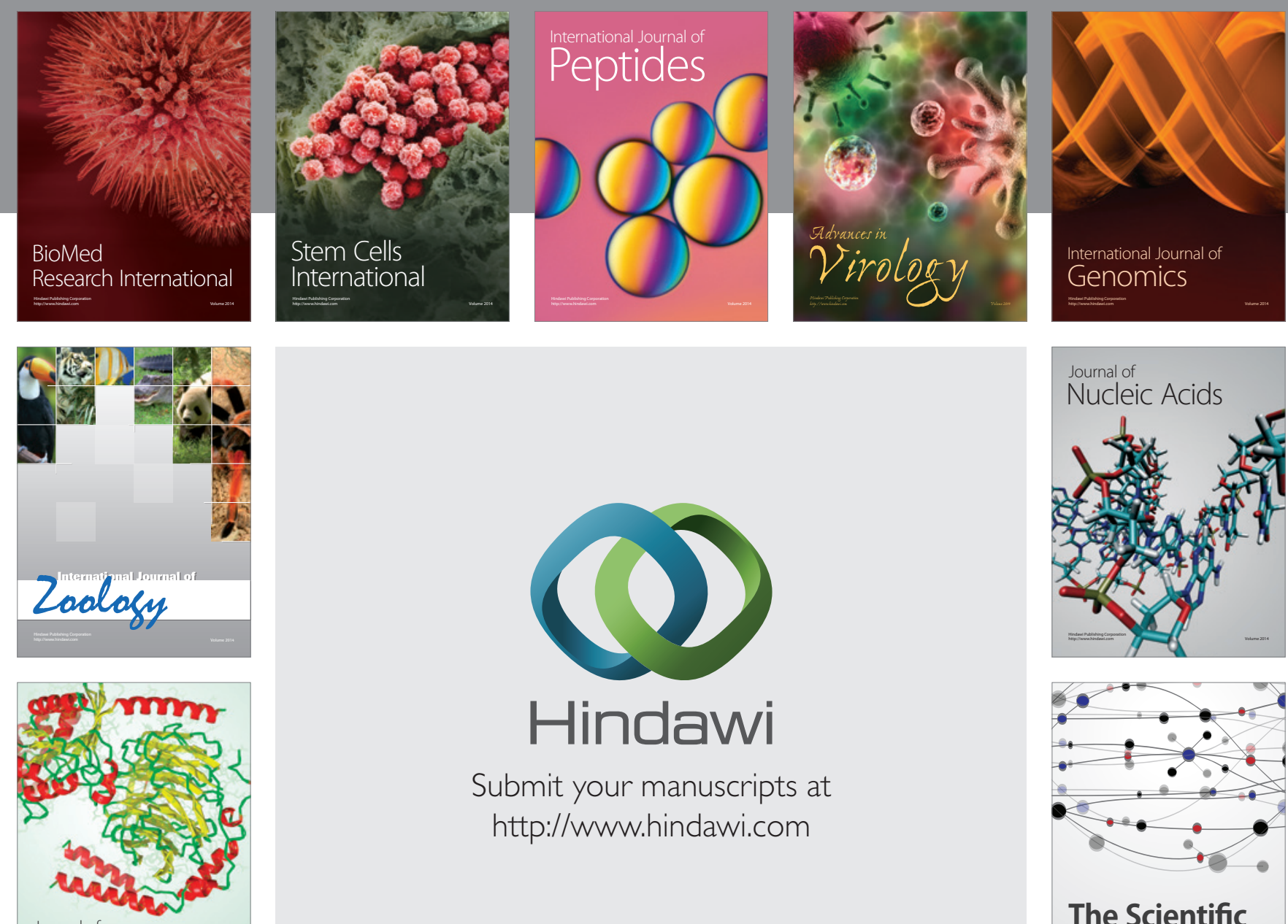

Submit your manuscripts at

http://www.hindawi.com

Journal of
Signal Transduction
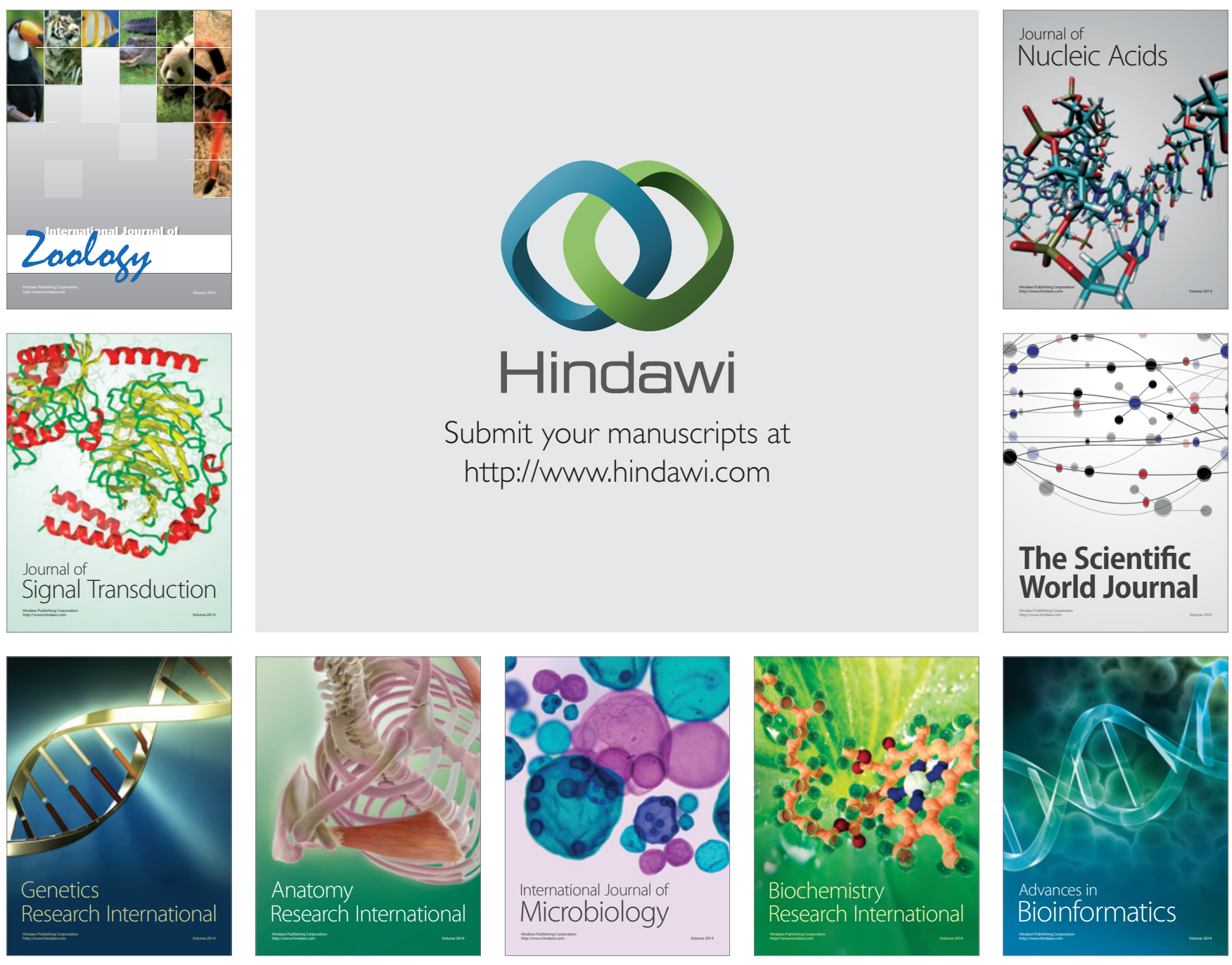

The Scientific World Journal
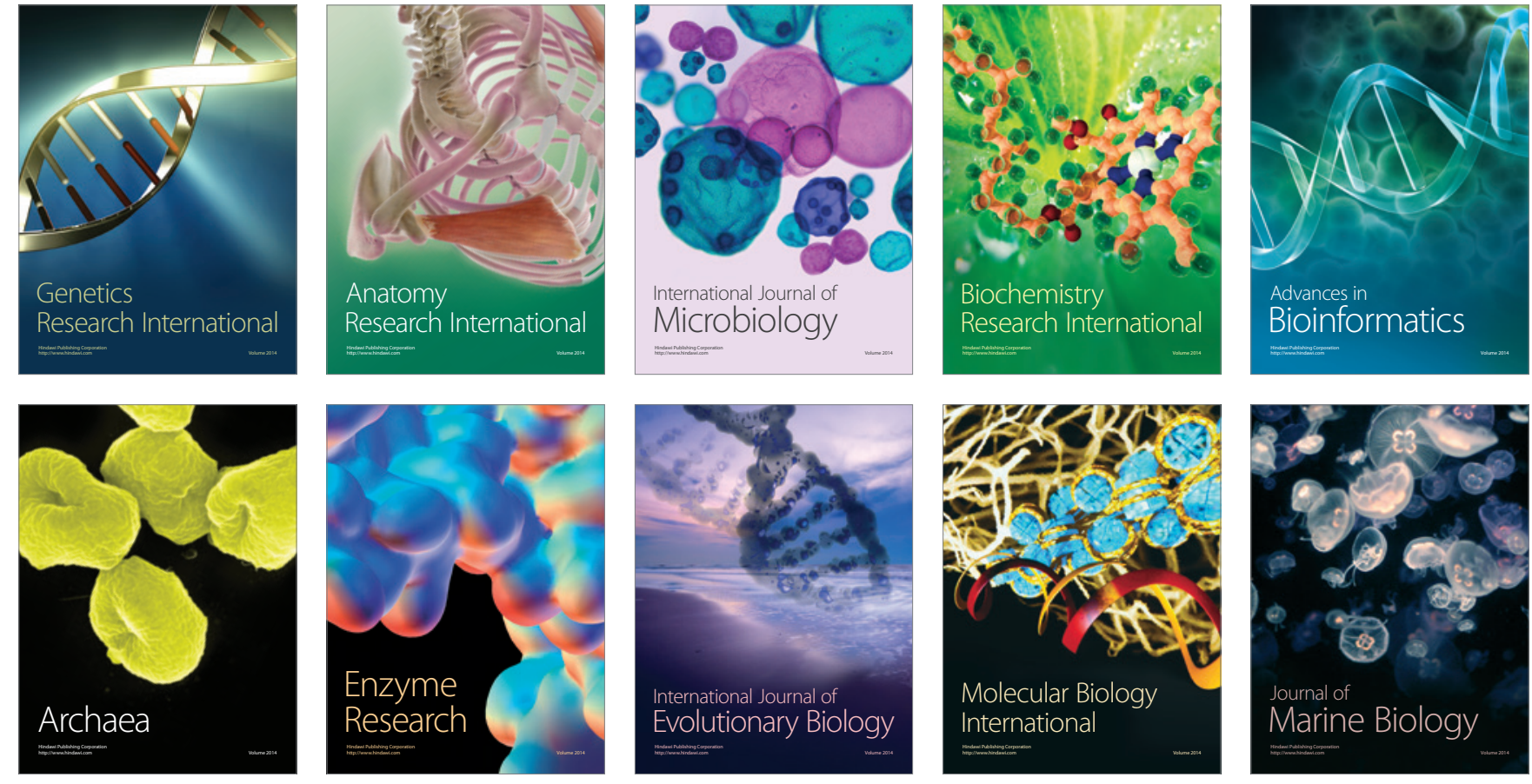